\title{
$\checkmark$ Research Square \\ A study in the effect of Oridonin on the lung cancer therapeutic effect of lentinan
}

\section{ZHIGUO CHEN ( $\nabla$ chenzhiguo8882@sina.com )}

Central Hospital of Wuhan

\section{Research Article}

Keywords: oridonin, lentinan, lung cancer, growth inhibition

Posted Date: May 3rd, 2021

DOI: https://doi.org/10.21203/rs.3.rs-488559/v1

License: (a) (i) This work is licensed under a Creative Commons Attribution 4.0 International License. Read Full License 


\section{Abstract}

Oridonin has been shown to have a potency for the improvement of the antitumor effect of lentinan (LNT). In this study, we tested the effect of oridonin, LNT, and the combination of them on a lung cancer. The study demonstrated that oridonin enhanced the antitumor effects of LNT and is conducive to the development of oridonin and LNT as a novel cancer drug regimen.

\section{Introduction}

In recent years, traditional medicine has been studied for clinical treatment for their low side effects $(1,2)$. To achieve fewer side effects, increasing attention has been given to a fungal polysaccharide, lentinan (LNT), for its strong antitumor activity $(3,4)$. It was reported to effectively affect division, differentiation, growth, and senescence of cells (5). LNT was reported to effectively prevent patients from cancers caused by chemical or viral carcinogens. In clinical, LNT enhances chemotherapy and improves the survival of patients in several cancer types, including gastric, colon, breast, and lung cancers. Current evidence also showed that it targeted small-cell lung cancer cells (6). Although it has a relatively weak effect on cancers, it is a promising chemotherapy synergist.

Recently, a compound from Rabdosia rubescens, oridonin, has been associated with cancer treatments. Studies showed that it suppressed breast cancer (7) and pancreatic cancer (8). It was also found to inhibit gene mutations induced by chemical carcinogens (9). It was also shown to block sodium pumps in cancer cells, decreased their nutrient intake (10), and regulated apoptosis and caspases pathways (11). A previous study showed that oridonin enhances the anticancer effects of lentinan in SMMC-7721 human hepatoma cells(12) and HepG2 human hepatoblastoma cells (13). However, its effect on lung cancer has not been studied.

Traditional medicine was used and studied previously (14). Some clinical findings in our hospital proposed that the use of oridonin enhances the treatment effects of LNT. In this study, human fetal lung fibroblast cell line MRC-5, lung cancer cell line A549, and Lewis lung carcinoma mice model were used to evaluate and validate the improvement of oridonin on the therapeutic effects of LNT on lung cancer.

\section{Materials And Methods}

\section{Cell lines}

Human fetal lung fibroblast cell line MRC-5, NSCLC cell line A549, and the Lewis lung carcinoma cells were obtained from the Conservation Genetics CAS Kunming Cell Bank (Kunming, Guangxi, China). RPMI1640 medium (Gibco BRL, Carlsbad, CA, USA) with 10\% fetal bovine serum (FBS; Gibco Co., Birmingham, MI, USA) was used to culture the cells. The cells were cultivated in the culture flasks under a humidified atmosphere containing $5 \% \mathrm{CO}_{2}$ at $37^{\circ} \mathrm{C}$.

\section{MTT assay}


The MTT method was described elsewhere (15).

\section{Real-time PCR assay}

The PCR method was described elsewhere (16).

\section{Western blotting}

The Western blotting method was described elsewhere (17).

\section{Experimental animal and Lewis lung carcinoma in vivo experiment}

C57BL/6J male mice at 7-weeks old were purchased from the Animal Center of the Wuhan University, which were fed at $23 \pm 1^{\circ} \mathrm{C}$ and $50 \pm 5 \%$ humidity with a 12-h light/dark cycle. The animal trials were approved by the Animal Ethics Committee of Wuhan University (Wuhan, Hubei, China). The modeling method was described elsewhere (18).

Lewis lung carcinoma was taken from liquid nitrogen and quickly put into $37^{\circ} \mathrm{C}$ water for melting, and then was centrifugated for $5 \mathrm{~min}$ at $14000 \mathrm{r} / \mathrm{min}$; the supernatant was abandoned, the remaining was diluted to $1 \times 10^{7} / \mathrm{ml}$. $0.2 \mathrm{ml}$ tumor cells suspension was subcutaneously injected into the right axillary of each C57BL/6J mouse. There were two sets of animal experiments, in each set, 10 mice without Lewis lung cancer were used as the normal group (without any cancer or treatment,). After the lewis lung cancer was induced in mice after 21 days, the mice were randomly divided into six groups, 18 mice for each group, control group (with cancer without treatment); LNT-L group $(0.2 \mathrm{ml} /$ day of $100 \mu \mathrm{g} / \mathrm{ml} \mathrm{LNT);}$ Oridonin group $(0.2 \mathrm{ml} /$ day of $20 \mu \mathrm{g} / \mathrm{ml}$ oridonin); oridonin + LNT-L group $(0.2 \mathrm{ml} /$ day of $20 \mu \mathrm{g} / \mathrm{ml}$ oridonin and $0.2 \mathrm{ml} /$ day of $100 \mu \mathrm{g} / \mathrm{ml} \mathrm{LNT})$; LNT-H group $(0.2 \mathrm{ml} /$ day of $300 \mu \mathrm{g} / \mathrm{ml}$ LNT); oridonin + LNT$\mathrm{H}$ group $(0.2 \mathrm{ml} /$ day of $20 \mu \mathrm{g} / \mathrm{ml}$ oridonin and $0.2 \mathrm{ml} /$ day of $300 \mu \mathrm{g} / \mathrm{ml}$ LNT). The dose was converted from the clinical dose for human patients. For the first set of animal experiments, after 10 days, the busts of the mice were measured with a mini tape measure before and after the treatment, and the bust increased over $50 \%$ compared with the size before injection was defined as significant lung cancer metastasis. All the mice were euthanized followed by the collection of lung tissues. The method of euthanasia used at end point was $\mathrm{CO}_{2}$ inhalation. The euthanasia chamber enabled animals to be readily visible which provided a minimum purity for $\mathrm{CO}_{2}$ of $99.0 \%$. This allowed unconsciousness with minimal distress to the animals. The lung tissues were used to extract mRNA and protein for the PCR and the Western Blotting assay. For the other set of experiments, the mice were fed until the endpoint to obtain the survival rate. The criteria for the endpoint were: 1) tumor growth that impedes the ability to ingest food or water; 2) the tumors pain or distress that cannot be relieved with palliative measures; 3) Solid tumors estimated to exceed $20 \%$ of normal body weight. The death of animals was recorded every 5 days.

\section{Statistical analysis}


All experiments were repeated at less three times. One-way ANOVA with post-hoc Tukey's tests was used to analyze the difference, $P<0.05$ was considered to indicate a statistically significant difference. SAS v9.1 statistical software package (SAS Institute Inc., Cary, NC, USA) was used for the statistics.

\section{Results}

\section{The effect of oridonin and LNT on the growth of MRC-5 and A549 cells}

Cell viabilities were determined using MTT assay. The viabilities of MRC-5 and A549 cells were not affected by $0-20 \mu \mathrm{g} / \mathrm{ml}$ oridonin. 0-300 $\mu \mathrm{g} / \mathrm{ml}$ LNT did not affect the viability of MRC-5 cells, but 50-400 $\mu \mathrm{g} / \mathrm{ml}$ LNT significantly inhibited the viability of A549 cells. The oridonin, LNT, or the combination of both did not affect MRC-5 cell viability. The oridonin had no effect on A549 cell viability but LNT significantly suppressed A549 cell viability, and the used of oridonin increased the suppression effect of LNT on A549 cells.

$20 \mu \mathrm{g} / \mathrm{ml}$ oridonin was used in the subsequent study because it was the highest concentration that had no significant effect on cell viability. In addition, $300 \mu \mathrm{g} / \mathrm{ml}$ LNT was selected for the subsequent study as a high concentration group (LNT-H) because it had the strongest potency in the viability of cancer cells while had no effect on the viability of normal cells. As a comparison, we also used a low concentration group (LNT-L) which was $100 \mu \mathrm{g} / \mathrm{ml}$ LNT.

\section{The effect of oridonin and LNT on mRNA and protein expression of apoptosis-associated proteins in} A549

LNT at both concentrations tested increased the mRNA and protein expressions of caspase-3, caspase-8, and caspase-9 in A549 cells with a larger increase in LNT-H. Oridonin further increased the mRNA and protein expression of caspase-3, caspase-8, and caspase-9 in LNT treated A549 cells. Besides, LNT at both concentrations tested increased the mRNA and protein expression of Bax in A549 cells with more increase in LNT-H (LNT high concentration group). Oridonin further increased the mRNA and protein expression of Bax in LNT treated A549 cells. On the other hand, LNT at both concentrations tested decreased the mRNA and protein expression of Bcl-2 and Bcl-xL in A549 cells with more decrease in LNT$\mathrm{H}$. Oridonin further decreased the mRNA and protein expression of Bcl-2 and Bcl-xL in LNT treated A549 cells. These results revealed that apoptosis might mediate the effects.

\section{The effect of oridonin and LNT on mRNA and protein expression of the p53/p21 pathway proteins in} A549

We suggested the viability effect was associated with p53/p21 signaling, thus we also tested them. LNT at both concentrations tested increased the mRNA and protein expression of p53 and p21 in A549 cells. Oridonin further increased the mRNA and protein expression of p53 and p21 in LNT treated A549 cells. These results suggested that the p53/p21 pathway was involved. 
Additionally, LNT at both concentrations tested increased the mRNA and protein expression of NF-KB in A549 cells with a more dramatic increase in LNT-H. Oridonin further increased the mRNA and protein expression of NF-KB in LNT treated A549 cells. On the other hand, LNT at both concentrations tested decreased the mRNA and protein expression of IKB- $\mathrm{a}$ in A549 cells with more decrease in LNT-H. Oridonin further decreased the mRNA and protein expression of IKB- $\alpha$ in LNT treated A549 cells. These results suggested that the NF-KB and IKB-a signaling was involved.

\section{The effects of oridonin and LNT on lung tumor metastasis in mice}

We established lung cancer metastasis mice model and treatment them with LNT-L, oridonin, oridonin + LNT-L, LNT-H, and oridonin + LNT-H. Mice without cancer or any treatment and mice with lung cancer but any treatment were used for comparison. After 10 days, the oridonin alone had no effect on short time lung cancer metastasis. LNT treatment decreased the metastasis with a higher inhibitory rate in LNT-H group than in LNT-L group. Oridonin improved the suppression of LNT against lung cancer metastasis in both groups. These results suggested that oridonin help LNT decrease lung tumor metastasis in mice.

\section{The effects of oridonin and LNT on the overall survival of mouse with lung tumor cells}

We also conducted a survival assay to test the effect of oridonin and LNT. Results showed that oridonin alone had almost no effect on the survival of the animals. Both LNT-L and LNT-H improved the survival and LNT-H had a better outcome than LNT-L. Oridonin increased the improvement effect of LNT on the survival rate. Results indicated that the combined use of both oridonin and LNT optimized survival.

\section{The effects of oridonin and LNT on the mRNA and protein expression of caspase-3, caspase-8, and caspase-9 in mice lung tissues}

After the lung cancer cell injection, the mRNA and protein expression of caspase-3, caspase-8, and caspase-9 significantly decreased in lung tissue samples. The LNT at both concentrations tested increased the mRNA and protein expression of caspase-3, caspase-8, and caspase- 9 in lung tissue samples with a more significant increase in LNT-H. Oridonin further increased the mRNA and protein expression of caspase- 3 , caspase- 8 , and caspase- -9 in the lung of LNT treated animals. These results were similar to those in A549, which confirmed the effect of oridonin and LNT on lung cancer.

\section{The effects of oridonin and LNT on the mRNA and protein expression of Bax, Bcl-2, and Bcl-xL in lung tissues}

In addition, after cancer induction, the mRNA and protein expressions of Bax significantly increased while $\mathrm{Bcl}-2$ and $\mathrm{Bcl}-\mathrm{xL}$ significantly decreased in lung tissue samples. LNT at both concentrations tested increased the mRNA and protein expressions of Bax while LNT-H increased more than LNT-L. Oridonin further increased the mRNA and protein expression of the expression of Bax in the lung. On the other hand, LNT at both concentrations tested decreased the mRNA and protein expressions of Bcl-2 and Bcl-xL with more decrease in the LNT-H group. Oridonin further decreased the mRNA and protein expressions of 
$\mathrm{Bcl}-2$ and $\mathrm{Bcl}-\mathrm{xL}$ in LNT treated groups. These results were similar to those in A549, which confirmed the effect of oridonin and LNT on lung cancer.

\section{The effects of oridonin and LNT on the mRNA and protein expression of p53 and p21 in mice lung tissues}

Besides, the cancer induction significantly decreased the mRNA and protein expressions of p53 and p21 in lung tissue samples. The LNT at both concentrations tested increased the mRNA and protein expressions of p53 and p21 in lung tissue samples with a more increased rate in LNT-H. Oridonin further increased the mRNA and protein expressions of p53 and p21 in the lung. These results were similar to those in A549, which confirmed the effect of oridonin and LNT on lung cancer.

\section{The effects of oridonin and LNT on the mRNA and protein expression of NF-KB and IKB-a in lung tissues}

Additionally, the mRNA and protein expression of NF-KB significantly increased while IKB-a significantly decreased in lung tissue samples. LNT at both concentrations tested decreased the mRNA and protein expression of NF-KB with more decrease in LNT-H. Oridonin further decreased the mRNA and protein expression of NF-KB in the lung of LNT treated animals. On the other hand, LNT at both concentrations tested increased the mRNA and protein expression of IKB-a with a larger increase in LNT-H. Oridonin further increased the mRNA and protein expression of ІкB- $\alpha$ in LNT treated lung tissues. These results were similar to those in A549, which confirmed the effect of oridonin and LNT on lung cancer.

\section{Discussion}

The significance of this work is to help explain the treatment effect of oridonin and LNT in clinical lung cancer patients in our hospital.

Our results showed that lung cancer cell line A549 was much more sensitive than normal human fetal lung fibroblast cell line MRC-5 toward LNT. This suggested that LNT can be a potential cancer medicine with low side effects. LNT was proved to have a suppression effect on some cancer cells (19) as well as an immunomodulatory effect on cancer patients (20). A retrospective study demonstrated that LNT improved the quality of cancer patients' life and remarkably promoted the efficacy of chemotherapy and radiation therapy during the cancer treatment (21) An in vivo study also revealed that LNT showed therapeutic potential for colitis-associated cancer. While for the oridonin, previous studies showed that 36 $\mu \mathrm{g} / \mathrm{ml}(0.1 \mathrm{mmol} / \mathrm{l})$ oridonin inhibited breast cancer growth and metastasis through blocking the Notch signaling (7).

In this study, we used the MTT assay to test the viability of lung cancer cells. The MTT assay was widely used in cancer pharmacological studies (22). Surprisingly, our results show that 0-300 $\mu \mathrm{g} / \mathrm{ml}$ oridonin had little effect on both MRC-5 and A549, indicating that lung cancer might have lower sensitivity than breast cancer toward oridonin. In addition, oridonin was also shown to inhibit human pancreatic cancer migration (8). The survival rate is a critical indicator of cancer therapy in different cancer types (23). 
Thus, we mimic the clinical treatment and survival in the mice as a previous study(24). The in vivo evidence of this study showed that oridonin along failed to suppressed the migration of lung cancer cells and did not affect the survival of mice with lung cancer. However, we found that oridonin promoted the effects of LNT both in the viability of A549 and the migration and the survival of mice. These results indicated that oridonin might trigger pathways that facilitate the actions of LNT. However, in survival experiment, we applied inhalation anesthesia. The anesthesia was reported to potentially affects cancers $(23,25)$, this might potentially affect the results.

To explore the underlying mechanisms, we tested several potential targets of LNT in both A549 and lung tissue samples. Studies show that proliferation and apoptosis affected cancer viability (26). We suggested that apoptosis might be involved in the effects of oridonin and LNT. Firstly, we looked at the caspase signaling pathway. Caspases, cysteine-aspartic proteases, cysteine-dependent aspartate-directed proteases, are a family of protease enzymes playing essential roles in programmed cell death and inflammation (27). A previous study showed that the co-treatment with paclitaxel and lentinan enhanced cell apoptosis rate by inducing caspase-3 activation (28). Here we discovered that LNT suppressed A549 cell viability by improving the expression of apoptosis executioner caspase-3 and the oridonin could further promote this improvement. Moreover, we also found potential negative feedback of the Caspases signal, since the expression of apoptosis initiator in Caspases, the Caspases-8 and 9, increased with Caspases-3.

In fact, lentinan was reported to exert synergistic apoptotic effects with paclitaxel in A549 cells (28). The apoptosis-inducing effect of lentinan was also reported in a study in human bladder cancer T24 cells (29). In hepatoma cells, oridonin was proved to promote the effects of lentinan through regulating apoptotic genes. To confirm that these effects are mediated by apoptosis (28), we further tested an apoptosis regulatory pathway, the Bax pathway. BCL2 family members act as anti- or pro-apoptotic regulators for cancer cells. Bcl-xL acts as an anti-apoptotic protein by preventing the release of mitochondrial contents such as cytochrome $c$, which leads to caspase activation and ultimately, programmed cell death (30). In the present study, the expressions of Bax, Bcl-2, and Bcl-xL were affected by LNT and oridonin, indicating that the effect of them was mediated by apoptosis.

The expression of the BCL2 family genes is regulated by the tumor suppressor p53 and has been shown to be involved in p53-mediated apoptosis $(31,32)$. We proposed that p53 and p21 might be the upstream targets of LNT and oridonin. Hence, the expression of p53 and p21 in A549 and lung tissues were determined, and it came out that the expressions of p53 and p21 were improved by LNT and oridonin. NF$\mathrm{KB}$, nuclear factor kappa-light-chain-enhancer of activated B cells, is a protein complex that controls transcription of DNA, cytokine production and cell survival while $1 \mathrm{kBa}$, nuclear factor of kappa light polypeptide gene enhancer in B-cells inhibitor alpha, is cellular proteins that function to inhibit the NF-KB transcription factors (33). The present study proved that NF-kB and IкBa are involved in the effects of LNT and oridonin on cancer, and their expression changed and played a role in the decrease of the cell viability. In the lung tissues, the result was similar to that of cancer cells, which confirmed the conclusion under in vivo condition. 
Our study demonstrated that oridonin enhanced the antitumor effects of LNT. We also found several potential regulatory targets of the effect of oridonin and LNT. However, there are other mechanisms that might be involved, such as cancer stem cells (34), ion channels in cancers(35-37), etc. Besides, studies have showed that traditional medicines(38) and anesthetics $(23,24,37)$ might affect cancer treatments. Hence, more work is required in the future. Recently, many compounds derived from traditional medicines were explored for potential clinical use (39-41). In Cancer treatment, although traditional medicines were not able to cure cancer alone, they are applied in the combination of medical therapy for reducing the adverse effects caused by chemotherapy or radiotherapy, thus improving therapeutic outcome and quality of life for patients (42). Here we tried to develop a compound from a traditional herb medicine to be a potential chemotherapy synergist. This study is conducive to the development of oridonin and LNT as a novel cancer drug regimen and contributes to the application of traditional medicine in clinical treatments.

\section{Declarations}

\section{Conflict of Interest}

There is no conflict of interest.

\section{References}

1. Liu $\mathrm{H}$, Xiong $\mathrm{Y}$, Wang $\mathrm{H}$, et al.: Effects of water extract from epimedium on neuropeptide signaling in an ovariectomized osteoporosis rat model. Journal of ethnopharmacology 221: 126-136, 2018.

2. Wang C, Chen G, Wang J, et al.: Effect of Herba Epimedium Extract on Bone Mineral Density and Microstructure in Ovariectomised Rat. Journal of Pharmaceutical and Biomedical Sciences 6, 2016.

3. Zhang $M$, Zhang $Y$, Zhang $L$ and Tian Q: Mushroom polysaccharide lentinan for treating different types of cancers: A review of 12 years clinical studies in China. Progress in molecular biology and translational science 163: 297-328, 2019.

4. Tian Y, Yi W, Bai L, et al.: Lentinan in-situ coated tungsten oxide nanorods as a nanotherapeutic agent for low power density photothermal cancer therapy. International Journal of Biological Macromolecules, 2019.

5. Meng $\mathrm{X}$, Liang $\mathrm{H}$ and Luo L: Antitumor polysaccharides from mushrooms: a review on the structural characteristics, antitumor mechanisms and immunomodulating activities. Carbohydrate research 424: 30-41, 2016.

6. Kidd PM: The use of mushroom glucans and proteoglycans in cancer treatment. Alternative medicine review 5: 4-27, 2000.

7. Xia S, Zhang X, Li C and Guan H: Oridonin inhibits breast cancer growth and metastasis through blocking the Notch signaling. Saudi pharmaceutical journal : SPJ : the official publication of the Saudi Pharmaceutical Society 25: 638-643, 2017. 
8. Gui Z, Luo F, Yang Y, Shen C, Li S and Xu J: Oridonin inhibition and miR200b3p/ZEB1 axis in human pancreatic cancer. Int J Oncol 50: 111-120, 2017.

9. Zhang W, Huang Q and Hua Z-C: Oridonin: A promising anticancer drug from China. Frontiers in Biology 5: 540-545, 2010.

10. WANG Z-n, WO X-d and ZHOU Y-I: Molecule mechanisms of Oridonin on tumors chemoprevention and therapy [J]. China Medical Herald 28, 2008.

11. Yang J, Jiang $\mathrm{H}$, Wang $\mathrm{C}$, et al.: Oridonin triggers apoptosis in colorectal carcinoma cells and suppression of microRNA-32 expression augments oridonin-mediated apoptotic effects. Biomedicine \& Pharmacotherapy 72: 125-134, 2015.

12. Xu T, Jin F, Wu K, Ye Z and Li N: Oridonin enhances in vitro anticancer effects of lentinan in SMMC7721 human hepatoma cells through apoptotic genes. Experimental and therapeutic medicine 14: 5129-5134, 2017.

13. Sun Z, Han Q, Duan L, Yuan Q and Wang H: Oridonin increases anticancer effects of lentinan in HepG2 human hepatoblastoma cells. Oncol Lett 15: 1999-2005, 2018.

14. Haixia W, Shu M, Li Y, et al.: Effectiveness associated with different therapies for senile osteoporosis: a network Meta-analysis. J Tradit Chin Med 40: 17-27, 2020.

15. Li R, Xiao C, Liu H, Huang Y, Dilger JP and Lin J: Effects of local anesthetics on breast cancer cell viability and migration. BMC cancer 18: 666, 2018.

16. Li X, Peng B, Zhu X, et al.: Changes in related circular RNAs following ERbeta knockdown and the relationship to rBMSC osteogenesis. Biochemical and biophysical research communications 493 : 100-107, 2017.

17. Liu X, Liu H, Xiong Y, et al.: Postmenopausal osteoporosis is associated with the regulation of SP, CGRP, VIP, and NPY. Biomedicine \& pharmacotherapy = Biomedecine \& pharmacotherapie 104: 742750, 2018.

18. Amikishieva AV, Ilnitskaya SI, Nikolin VP, Popova NA and Kaledin VI: Depressive-like psychoemotional state versus acute stresses enhances Lewis lung carcinoma metastasis in C57BL/6J mice. Experimental oncology 33: 222-225, 2011.

19. Qian Y, Wang D, Fan M, Xu Y, Sun X and Wang J: Effects of intrinsic metal ions of lentinan with different molecular weights from Lentinus edodes on the antioxidant capacity and activity against proliferation of cancer cells. International journal of biological macromolecules 120: 73-81, 2018.

20. Wang X-e, Wang Y-h, Zhou Q, et al.: Immunomodulatory Effect of Lentinan on Aberrant T Subsets and Cytokines Profile in Non-small Cell Lung Cancer Patients. Pathology \& Oncology Research: 1-7, 2018.

21. Zhang M, Zhang Y, Zhang L and Tian Q: Mushroom polysaccharide lentinan for treating different types of cancers: A review of 12 years clinical studies in China. Progress in molecular biology and translational science 163: 297-328, 2019.

22. Hengrui Liu JPD, Jun Lin: Effects of local anesthetics on cancer cells. Pharmacology \& Therapeutics in press, 2020. 
23. Li R, Liu H, Dilger JP and Lin J: Effect of Propofol on breast Cancer cell, the immune system, and patient outcome. BMC anesthesiology 18: 77, 2018.

24. Li R, Huang Y, Liu H, Dilger JP and Lin J: Comparing volatile and intravenous anesthetics in a mouse model of breast cancer metastasis. American Association for Cancer Research, p 2162, 2018.

25. Liu H: A clinical mini-review: Clinical use of Local anesthetics in cancer surgeries. The Gazette of Medical Sciences 1: 030-034, 2020.

26. Liu H, Dilger JP and Lin J: The Role of Transient Receptor Potential Melastatin 7 (TRPM7) in Cell Viability: A Potential Target to Suppress Breast Cancer Cell Cycle. Cancers 12, 2020.

27. Van Opdenbosch $\mathrm{N}$ and Lamkanfi M: Caspases in Cell Death, Inflammation, and Disease. Immunity 50: 1352-1364, 2019.

28. Liu W, Gu J, Qi J, et al.: Lentinan exerts synergistic apoptotic effects with paclitaxel in A549 cells via activating ROS-TXNIP-NLRP3 inflammasome. Journal of cellular and molecular medicine 19: 19491955, 2015.

29. Bao L, Wang Y, Ma R, Ren X, Cheng R and B A: Apoptosis-inducing effects of lentinan on the proliferation of human bladder cancer T24 cells. Pakistan journal of pharmaceutical sciences 28 : 1595-1600, 2015.

30. Siddiqui WA, Ahad A and Ahsan H: The mystery of BCL2 family: Bcl-2 proteins and apoptosis: an update. Archives of toxicology 89: 289-317, 2015.

31. Wawryk-Gawda E, Chylińska-Wrzos P, Lis-Sochocka M, et al.: P53 protein in proliferation, repair and apoptosis of cells. Protoplasma 251: 525-533, 2014.

32. Wang X, Simpson ER and Brown KA: p53: protection against tumor growth beyond effects on cell cycle and apoptosis. Cancer research 75: 5001-5007, 2015.

33. DiDonato JA, Mercurio F and Karin M: NF-KB and the link between inflammation and cancer. Immunological reviews 246: 379-400, 2012.

34. Liu H: A Prospective for the Potential Effect of Local Anesthetics on Stem-Like Cells in Colon Cancer. Biomedical Journal of Scientific \& Technical Research 25: 18927-18930, 2020.

35. Liu H: Nav channels in cancers: Nonclassical roles. Global Journal of Cancer Therapy 6: 5, 2020.

36. Liu H: A prospective for the role of two-pore channels in breast cancer cells. Global Journal of Cancer Therapy 6: 001--003, 2020.

37. Liu H, Dilger JP and Lin J: Lidocaine Suppresses Viability and Migration of Human Breast Cancer Cells: TRPM7 as A Target for Some Breast Cancer Cell Lines. Cancers 13: 234, 2021.

38. Liu H: Effect of Traditional Medicine on Clinical Cancer. Biomedical Journal of Scientific \& Technical Research 30: 23548-23551, 2020.

39. Wu Z, Ou L, Wang C, et al.: Icaritin induces MC3T3-E1 subclone14 cell differentiation through estrogen receptor-mediated ERK1/2 and p38 signaling activation. Biomedicine \& pharmacotherapy $=$ Biomedecine \& pharmacotherapie 94: 1-9, 2017. 
40. Chen G, Wang C, Wang J, et al.: Antiosteoporotic effect of icariin in ovariectomized rats is mediated via the Wnt/beta-catenin pathway. Experimental and therapeutic medicine 12: 279-287, 2016.

41. Liu H, Xiong $Y$, Zhu X, et al.: Icariin improves osteoporosis, inhibits the expression of PPARgamma, C/EBPalpha, FABP4 mRNA, N1ICD and jagged1 proteins, and increases Notch2 mRNA in ovariectomized rats. Experimental and therapeutic medicine 13: 1360-1368, 2017.

42. Xiang Y, Guo Z, Zhu P, Chen J and Huang Y: Traditional Chinese medicine as a cancer treatment: Modern perspectives of ancient but advanced science. Cancer Med 8: 1958-1975, 2019. 